\title{
Urgences
}

\section{De la terre visible aux terres de l'invisible}

\section{Claude Mettra}

Numéro 17-18, octobre 1987

L'esprit des lieux

URI : https://id.erudit.org/iderudit/025421ar

DOI : https://doi.org/10.7202/025421ar

Aller au sommaire du numéro

Éditeur(s)

Urgences

ISSN

0226-9554 (imprimé)

1927-3924 (numérique)

Découvrir la revue

Citer ce document

Mettra, C. (1987). De la terre visible aux terres de l'invisible. Urgences, (17-18),

66-73. https://doi.org/10.7202/025421ar d'utilisation que vous pouvez consulter en ligne.

https://apropos.erudit.org/fr/usagers/politique-dutilisation/ 


\section{Deuxième atelier: 2 juin: 19 h30 \\ Claude Mettra \\ Raymond Montpetit \\ Paul Chanel Malenfant}




\section{Claude Mettra \\ DE LA TERRE VISIBLE \\ AUIX TERRES DE L'INVISIBLE}

Si nous avions pu convier Socrate à notre rencontre, il eut sans doute commencé par invoquer Pan, dieu de la vie sauvage et de l'extase amoureuse mais surtout dieu de la musique. Car comme nous le murmure Platon, se référant ici à la tradition pythagoricienne, c'est peut-être à travers la musique qu'il nous est possible de sentir la trace de cet esprit qui cherche son lieu en nous ou autour de nous. Charles-Ferdinand Ramuz a appelé l'un de ses romans La beauté sur la terre, et cette beauté, la plupart du temps sans visage, nous bouleverse jusqu'au fond du coeur parce qu'elle est comme le signe de cet esprit qui nous cherche mais reste si souvent hors de notre portée.

Le lieu, nous savons ce qu'il est. Dans le langage commun, nous disons que nous sommes perdus, égarés, pour indiquer que nous sommes comme des naufragés sur une mer inconnue et déchaînée; semblables aux petits enfants des contes de fées, nous marchons à travers la forêt mystérieuse sans parvenir à trouver l'enchanteur. Et cependant nous sommes sûrs que quelque part il existe un château construit à notre mesure, une demeure où l'esprit est prêt à nous accueillir.

Je voudrais ici évoquer un roman de Michaël Ende: L'Histoire sans fin. On y raconte l'histoire d'un petit garçon qui s'appelle Bastien. Il a à peu près douze ans̀ et, dans ces circonstances assez bizarres, il est projeté dans un monde de l'ailleurs. Là, sa mission est de venir au secours de la souveraine de ce royaume de l'ailleurs, une petite princesse qui a perdu son nom; seul un enfant des hommes peut retrouver le nom de cette princesse et ainsi sauver ce royaume qui, au fur et à mesure que le temps passe, se défait exactement comme se défait un nuage.

Ce petit garçon va donc redécouvrir le nom de la petite princesse Lune. Et après, il lui faut retrouver son espace terrestre, sa demeure, son pays. Il erre longtemps, il ne sait pas trop où est le mot de passe qui lui permettra de regagner son domaine. Un jour, il arrive dans une grande plaine neigeuse. Il aperçoit dans cette plaine une petite hutte vers laquelle il se dirige et, là, il y a un vieux mineur, et ce mineur est aveugle. Bastien lui dit: "qu'est-ce que tu fais là?", et l'homme lui répond: «demain matin, tu viendras avec moi». Et le lendemain l'enfant descend avec lui dans la mine; à l'intérieur de la 
terre, cet homme découpe délicatement de grandes plaques mystérieuses qui sont un peu comme des plaques de mica, comme des vitraux, qu'il met dans sa benne. Puis, quand il en a récolté un grand nombre, il les ramène à la surface de la terre et va les disposer les unes à côté des autres dans la neige. Bastien est très intrigué par ce jeu. Ces vitraux racontent des histoires étranges et le mineur lui dit: "Ce sont les rêves de tous les humains; vous autres les hommes, vous rêvez et après, vous oubliez vos rêves. Mais ces rêves ont besoin de vivre, alors ils se réfugient à l'intérieur de la terre. Ils sont là ainsi, tous étalés les uns à côté des autres, et il faut faire très attention à ne pas les briser parce que, s'ils étaient brisés, ceux qui ont eu ces rêves en seraient quelque peu blessés. Et toi, Bastien, tu dois trouver parmi tous ces rêves celui qui est le tien: c'est ce qui te permettra de revenir sur la terre». Et finalement Bastien retrouvera cette image et son domaine.

Comme cet enfant, chacun d'entre nous est appelé à reconnaître cette image ou ces images premières qui constituent le lieu originel de notre coeur et dont nous entrevoyons le double dans notre vie réelle. Car l'émerveillement d'être au monde commence peut-être au moment où la demeure de l'âme se confond avec la demeure de notre incarnation. Mais que nous disent les mythes à ce propos?

\section{Jouissance, connaissance et création}

Dans La Théogonie, Hésiode raconte qu’au commencement fut l'abîme, le chaos. Un monde de l'informe semblable à celui que nous ressentons parfois quand nous ne nous sentons pas dans notre lieu, quand nous ne sommes pas dans notre peau ou que nous y sommes mal. Du chaos naquit la Terre, Gaia, à l'assise sûre; la terre aux larges flancs, ce qui évoque immédiatement l'idée de la fécondité. Face à cette terre va apparaître la Nuit. Ainsi s'annonce cette dramaturgie dont nous sommes toujours les témoins, à la fois en dehors de nous et en nous. Et toute l'histoire, celle des autres et la nôtre, va s'écrire dans cet antagonisme entre les énergies de la vie et les forces de l'obscurité et de la mort.

Gaia va faire accéder à la vie la multitude des formes dont nous partageons l'aventure: celles du ciel, celles de la terre et celles des mondes inférieurs. Et cette volonté créatrice, telle que la dépeint Hésiode, s'accomplit avec l'aide d'Eros, principe du désir, sous trois visages majeurs. D'abord celui de la jouissance, car la vie est joie, et cette jouissance, selon l'expression d'Alain Daniélou, est la substance de la vie. Et pour accéder à ce plaisir d’amour, Gaia va créer de son propre sein, son compagnon, Ouranos, le ciel étoilé. 
Au delà de cette soif de plaisir, il y a la volonté de connaissance qui se manifeste à travers la création des luminaires et des astres, c'est-à-dire de ce qui peut éclairer le monde.

Enfin le troisième aspect de cette entreprise pour être et pour exister, c'est le désir de fécondité. De son étreinte avec Ouranos, qui se reproduit chaque nuit comme si l'amour était là pour exorciser les forces des ténèbres, vont naître de multiples et terribles enfants dont la mythologie nous raconte inlassablement les cruels ou triomphants destins.

Et ce qui est mis en scène par Hésiode, c'est la toute puissance de l'imagination, la possibilité pour Gaia de fonder le tout à partir de rien; et pour nous, par référence à cette Mère première, la possibilité de créer l'infini à partir du fini, l'invisible à partir du visible.

Et là est peut-être le secret de notre relation avec l'esprit des lieux. Il y a d'un côté le coeur de la terre, et de l'autre notre propre coeur. De leur rencontre peut naître ce dialogue amoureux qui, comme dans nos amours humaines, va révéler l'homme à la terre et la terre à l'homme. C'est notre esprit qui crée le lieu mais c'est aussi le lieu qui, en nous, crée l'esprit. Mais où donc se situe la source mystérieuse de cette rencontre? Où se manifeste cette confluence entre l'eau de la vie et le sang des hommes?

\section{La main de l'oubli}

Certains textes gnostiques, dont on retrouve trace beaucoup plus tard dans la tradition hassidique, évoquent celui que j'appellerai ici l'Ange de la naissance et qui sans doute n'est pas sans lien avec l'Ange gardien du christianisme tardif. Il existe, pour reprendre l'expression du romancier scandinave Pär Lagerkvist, un pays des âmes, un grand lac de semence où les êtres participent de la lumière et de la béatitude divine. Mais l'archonte suprême ordonne de temps à autre à telle ou telle de ces âmes de s'incarner et il charge l'Ange de mener l'oeuvre à son terme, car bien entendu l'âme ressent devant cette incarnation angoisse et regrets. Bien malgré elle, la voilà condamnée à trouver place dans le ventre de la femme.

Au moment de la naissance, et nous retrouvons ici l'image du fleuve Léthé familier à la Grèce, l'Ange se tient à la porte du ventre maternel et lorsque l'enfant apparaît à la clarté du jour, cet ange étend la main pour effacer tout souvenir de l'existence antérieure car, comment l'être humain pourrait-il parcourir son aventure terrestre s'il avait mémoire vivace du lieu paradisiaque où il lui avait été donné de vivre jadis? Son coeur serait indéfiniment déchiré par la nostalgie de l'ailleurs. Il ne serait plus qu'une créature pour le deuil. 
Mais comme l'indiquent à l'abondance les mythologies et les récits fondateurs, que ce soit la Bible ou les Upanishads, les êtres célestes (divinités, anges ou héros immortels) sont comme nous la proie de forces, d'énergies ou de désirs dont ils ne sont pas les maîtres. Ou bien ils se meuvent dans des horizons qui n'obéissent pas aux mêmes lois que les nôtres. Et cet Ange de la naissance qui de sa main doit effacer toute mémoire ancienne, voilà que de temps à autre il n'étend pas la main comme il le faudrait. Est-ce par distraction, tout entier qu'il est à la pensée de la musique qui est son occupation favorite, si du moins l'on en croit deux millénaires d'iconographie chrétienne; ou bien est-ce par ironie, et comme pour imprimer sa marque sur le devenir de la création? Ou bien est-ce délibérément qu'il décide de laisser à l'âme souvenance d'une part de son lac perdu, confiant ainsi à un destin privilégié le soin de maintenir une certaine circulation entre le pays des âmes et le pays des corps, entre l'infini de l'espace céleste et le fini de l'espace terrestre? Qui sait?

On pourra bien entendu relier cette figure angélique aux perspectives développées par Ferenczi dans Thalassa et à son évocation du paradis de nature océanique qui représenterait le ventre maternel. Le psychanalyste hongrois réduit à la mesure humaine, à l'espace concret de la mère ce qui pour les gnostiques ne saurait se concevoir que sur le plan de l'espace spirituel. On notera aussi que cette main de l'Ange semble reproduire à sa manière le mouvement de la main de Dieu quand il façonne l'homme à partir de l'argile.

J'ai dépeint l'Ange se hâtant ainsi vers cette porte qui ouvre le ventre de la mère mais on peut imaginer aussi que l'Ange étend la main au moment même de la conception et qu'ainsi dès le commencement de son incarnation, l'être humain est séparé de son existence antérieure. La question est d'importance, même aux yeux d'une psychologie contemporaine qui s'intéresse de plus en plus vivement à la manière dont l'enfance traverse les neuf mois de son élaboration. Et le paradis océanique qu'évoque Ferenczi, cette eau de nature marine où le petit baigne dans son temps d'obscurité, est-ce qu'il est, ou non traversé par les imageries de l' «arrière-pays»? Si c'est le cas, le rôle de la mère, qui laisse ou fait vivre en elle ce trésor d'avant la vie, se trouve singulièrement conforté, celui du père n'étant plus que l'élément prétexte de la naissance.

\section{La matrice des rêves}

J'ai parlé du geste inachevé de l'Ange. Et l'intuition première de cet événement, je l'ai eue dans un vaste parcours accompli à travers la littérature romantique et plus particulièrement la littérature alle- 
mande. Chez Novalis, chez Ludwig Tieck, plus tardivement chez Adalbert Stifter ou Gottfried Keller, sont rapportés de nombreux rêves enfantins. Ces rêves constituent toujours des expériences fondamentales pour ceux qui en sont le territoire, et bien au delà de l'âge enfantin, ils reviennent, toujours actifs, toujours fécondants jusque dans l'âge vieillissant.

Ces rêves ne sont pas réductibles à ce qui est le champ de la psychologie d'aujourd'hui. Ils ne sont pas les témoins plus ou moins travestis, plus ou moins indéchiffrables d'événements réellement vécus. Ils apparaissent bien plutôt comme les vestiges un peu délabrés d'une expérience beaucoup plus ancienne, celle sans doute à laquelle se réfère Henri Michaux lorsqu'il écrit «L'enfant, l'infini, il sait ce que c'est, il en vient". J'assimilerais volontiers ces rêves aux visions que nous proposent les grands textes mystiques comme ceux d'Hadewijch d'Anvers ou de Sainte Thérèse, mais on peut songer aussi aux Illuminations d'Arthur Rimbaud.

Pour retrouver dans notre propre coeur, ce qui peut-être demeure en nous de puissance visionnaire, de trace de notre aventure cosmique, force nous est de descendre à l'intérieur de ce que John Cooper Powys appelle notre puits de granit, ce lieu originel délivré de toutes les souillures dont l'histoire, la nôtre, mais aussi celle de notre communauté, nous a chargés. C'est de cette démarche que rend compte le roman de Novalis Henri d'Ofterdingen. Le poète met ici en scène un adolescent qui a gardé en lui une part de l'innocence enfantine. Dans son village vient un jour un vieil homme, un errant nullement misérable qui raconte une histoire où il est question d'une fleur bleue.

Dans la nuit qui suit sa rencontre avec le vieil homme, Henri fait un rêve. Il traverse de multiples paysages à la fois tourmentés et radieux. Puis il arrive près d'une cascade autour de laquelle il y a de splendides papillons et des libellules. Et, dans son rêve, il demande aux papillons: "Où est la demeure d'Isis?» c'est-a-dire la Mère éternelle. Et les papillons lui disent: "Continue simplement ton chemin". Il parvient alors au bord d'un lac qu'il traverse; au milieu du lac il y a une île et dans cette île une fleur exactement semblable à celle dont le vieil homme lui avait parlé la veille. Il se penche délicatement sur la fleur et la corolle s'ouvre lentement; apparaît alors le visage d'une jeune fille, de cette jeune fille dont, lui semble-t-il, il n'a cessé de rêver.

C'est sur cette image qu'Henri s'éveille. Et la suite du roman n'est que le récit du voyage aventureux qui conduira l'adolescent jusqu'à la rencontre avec une jeune fille semblable à celle dont la fli: 
bleue lui avait révélé le visage. Mais cette rencontre n'ira pas sans une série d'initiations dont le thème commun est la descente dans la mine, c'est-à-dire dans la terre qui est l'image de notre propre coeur.

Le premier maître de ce parcours initiatique est en effet un homme qui, après une longue expérience de la mine, a choisi de vivre dans les entrailles de la terre. Il fait songer au philosophe de Rembrandt méditant dans le clair obscur de la cave. Il invite Henri à l'accompagner dans ses domaines mystérieux, là où il n'y a plus d'autre parole que celle du souterrain. Et l'on ne peut s'empêcher de songer ici aux initiations pythagoriciennes. Le néophyte qui voulait partager la vie de la communauté devait rester enfermé trois jours et trois nuits dans la solitude de la caverne. Cette caverne devenait véritablement le lieu où l'esprit pouvait se manifester. Et c'est quand Henri, à la suite de divers périples dont la terre intérieure est le théâtre, aura rencontré à la fois l'âme secrète du monde et sa propre vérité, qu'il pourra reconnaître le visage vivant et impérissable de l'amour.

\section{La parole de l'être}

Mais quelle relation pouvons-nous donc établir entre les rêves révélateurs de l'Arrière-Pays et la caverne où nous convient Pythagore et Novalis? Ni la parole des rêves ni celle de la terre ne nous sont clairement déchiffrables. Vainement nous nous efforcerions de les enfermer dans la logique de notre quotidien. Comment donc définir ce qui est proprement, selon le thème de notre rencontre, l'esprit des lieux? Car l'esprit est constamment présent dans les rêves comme il est constamment présent dans le silence de la terre. Et il est à la fois lumière et son. Vocable que l'on retrouve d'ailleurs dans le langage amoureux puisque l'on dit de ceux qui s'aiment qu'ils vibrent à l'unisson, mot singulier que l'on emploie, je crois, en cette seule circonstance. Et l'on pourrait aisément montrer que lorsque l'on parle, dans une perspective où l'affectif est en jeu, de vibration, il est difficile de dissocier ce qu'il y a en elle de lumineux et de sombre. Je pourrais évoquer ici aussi bien Rembrandt que Bach. Les figurations plastiques du peintre d'Amsterdam nourrissent en nous une rêverie proprement musicale et les cantates du maître de chapelle de Leipzig sont liées originellement aux rayonnements de la lumière à travers les vitraux des cathédrales.

Mais cette expérience de là vibration, chacun la peut retrouver aisément sans descendre dans la caverne, simplement dans la marche... Tous les grands marcheurs savent qu'en cette simple relation avec la terre s'accomplit une profonde métamorphose en notre être. Car tout se passe comme si la terre, lentement, prenait en charge notre propre tourment, nos humbles soucis. Elle nous récom- 
pense ainsi de poser sur elle un pas qui lui est caresse. Il y a un échange d'ordre amoureux entre la glèbe et nous; c'est à travers cet échange que nous nous trouvons, au sens alchimique du terme, purifiés. Et quand cette relation d'affection est profonde, durable, ou bien lorsque d'instinct nous reconnaissons en une terre le reflet de notre propre coeur, le lieu devient proprement magique, habité d'esprit.

Nous cessons alors d'être enfermés dans notre carapace charnelle pour devenir un fragment du souffle cosmique; nous participons, dans un état de non-séparation, au mouvement de la vie.

Cette relation lumineuse et sombre avec la création et avec le monde nous renvoie aux grands mythes fondateurs communs à l'Orient et à l'Occident. L'invention du monde commence soit avec l'apparition de la lumière, soit avec le son primitif comme l'a étudié le grand musicologue allemand Marius Schneider dans le chapitre qu'il a consacré à la mythologie et aux rites dans L'Histoire de la Musique de l'Encyclopédie de la Pléiade.

Et ce que semblent révéler à la fois nombre d'expériences poétiques et de multiples témoignages que nous proposent aujourd'hui les recherches psychologiques, c'est qu'il existe peut-être pour chacun d'entre nous une vibration particulière; il faut parfois une existence d'homme toute entière pour la découvrir ou du moins la pressentir. Et je crois qu'un certain nombre d'émotions bouleversantes, apparemment sans objet ni cause apparente, sont liées à une approche de cette vibration. Ce que nous pouvons ressentir devant un paysage, devant un visage, devant une image ou dans la seule écoute du vent ou des vagues...

C'est pourquoi d'ailleurs certaines rencontres fugitives nous plongent parfois dans une profonde mélancolie dont nous ne parvenons pas à saisir la source. Notre inconscient murmure qu'il y a là une parole qui nous concerne, un signe qui nous est adressé, mais tout nous reste obscur. Nous allons, et là est la nature vraie de la mélancolie, avec le sentiment d'avoir perdu quelque chose mais nous ne savons pas quoi. Et nous serons condamnés à passer des saisons et des ans à chercher un trésor dont nous ignorons tout, mais, comme le dit Novalis, «l'essentiel n'est pas le but mais le chemin". La "Terre gaste» qu'évoquent les romans de la matière de Bretagne représente ce continent désolé auquel les quêteurs du Graal restitueront un jour sa grâce.

Cette jonction entre l'harmonie du coeur et l'harmonie du 
monde, Nietzsche consacra sa vie à la retrouver. L'image de l'Eternel Retour est peut-être le symbole de ce temps promis à chacun d'entre nous où enfin nous deviendrons perceptibles à la musique et à la lumière de notre âme. Entreprise qui ne va pas sans péril. Dans son dernier message à son ami Peter Gast, Nietzsche s'écria: «Adieu, j'ai vu plus loin qu'il n'est permis". Mais cette plongée dans le secret du monde est sans doute le moyen qui nous est donné d'aider la création à s'accomplir, la terre à perpétuellement ressusciter. Et dans cette aventure nous ne sommes ni dans le passé, ni dans le futur, ni dans le présent. Nous sommes simplement des enfants de l'infini. 